\title{
A catalogue of the collections of Mexican amber at the Natural History Museum, London and National Museums Scotland, Edinburgh, UK
}

\author{
Andrew J. Ross ${ }^{1,2 . *}$, Claire J.T. Mellish ${ }^{2}$, Bill Crighton ${ }^{1}$, Peter V. York ${ }^{3}$ \\ ${ }^{1}$ Department of Natural Sciences, National Museum of Scotland, Chambers St., Edinburgh, EH1 1JF. \\ ${ }^{2}$ Department of Earth Sciences, Natural History Museum, Cromwell Road, London, SW7 5BD. \\ ${ }^{3}$ Department of Life Sciences, Natural History Museum, Cromwell Road, London, SW7 5BD. \\ *a.ross@nms.ac.uk
}

\begin{abstract}
A catalogue is here provided of the pieces of Mexican amber with inclusions in the collections of the Natural History Museum, London, and National Museums Scotland, Edinburgh, both in the United Kingdom. There are 32 pieces in the Natural History Museum and 101 pieces in National Museums Scotland which contain a combined total of 557 arthropod and 13 plant inclusions. Four orders and 11 families of arthropods are additional to a taxonomic list of Mexican amber arthropods published in 2010.
\end{abstract}

Keywords: Mexican amber, inclusions, arthropods, London, Edinburgh.

Resumen

Presentamos aquí el catálogo de piezas de ámbar de México con inclusiones encontrados en las colecciones del Museo de Historia Natural de Londres y del Museo Nacional de Escocia en Edimburgo, ambos en el Reino Unido. En el Natural History Museum encontramos 32 piezas, mientras que, en el Museo Nacional de Escocia, encontramos un total de 101 piezas. Se incluye un total de 557 artrópodos y 13 inclusiones de plantas. Cuatro órdenes y 11 familias de artrópodos son adicionales a la lista taxonómica mexicana de artrópodos en ámbar publicada en el 2010.

Palabras clave: ámbar de México, inclusiones, artrópodos, Londres, Edimburgo.

\section{Introduction}

For the past 20 years the Natural History Museum in London (NHM) and National Museums Scotland in Edinburgh (NMS) have been acquiring specimens of Mexican amber with inclusions by purchase or donation. This paper comprises a list of the specimens to make the wider scientific community aware of this material and to hopefully generate research on the specimens.

There are currently 32 pieces of Mexican amber with inclusions in the collection of the NHM and 101 pieces in NMS. Many of these pieces contain several inclusions and contain a combined total of 557 arthropods and 13 plant remains (although one may be fungal).

Most of the specimens have not been studied in detail so the identifications were predominantly undertaken by AJR. Over the years various visiting scientists have improved some of the IDs and a few new taxa have been described.

\section{Catalogue}

The catalogue below is organised by major taxonomic group (in capitals), then by order and family where possible. 
Order and family names of arthropods given in bold were not included in Solórzano Kraemer (2010), of which there are four additional orders and eleven additional families listed here. For those where a family ID has not yet been provided then 'Undetermined' is used though some of these specimens may be fragmentary or poorly preserved and so are indeterminate. Specimen numbers pre-fixed by a question mark show that the identification is tentative. The total number of inclusions is given in brackets after the taxonomic name, excluding trace fossils. Further identifications and type details are also provided.

Twenty four specimens are figured here, including previously described holotypes. Fourteen of these specimens have not been previously figured. The corresponding figure number is given in brackets, in bold, after the specimen number. All scale bars represent $1 \mathrm{~mm}$.

The specimens at the NHM are pre-fixed by II (for insects), IA (for arachnids) or V (for plants). The main three or four-digit number is the number of the piece of amber; the number in brackets denotes the number for the taxon where more than one taxon occur in the one piece; if there is more than one specimen of that taxon in the amber then the quantity is given in brackets, e.g. II.2132(2) (x2) denotes amber piece with insects no. 2132, taxon no. 2 and $\mathrm{x} 2$ specimens of that taxon.

The specimens at the NMS are prefixed with $\mathrm{G}$ followed by the year of acquisition, the batch number, the number of the amber piece, the number of the taxon if there is more than one, and the quantity of that taxon is given in brackets if more than one specimen exists, e.g. G.2004.6.10.1 (x3) denotes year of acquisition 2004 , batch no. 6 , amber piece no. 10 , taxon no. 1 and $\mathrm{x} 3$ specimens of that taxon. The specimens are kept at the National Museums Collection Centre in Edinburgh, which is part of National Museums Scotland.

The catalogue was compiled by AJR and CJTM, with photographs of NMS specimens by BC (Copyright NMS) and NHM specimens by PVY (Copyright NHM), unless otherwise stated in the figure caption.

\section{ARACHNIDA (52)}

Acari (3):

Undetermined (3):

- $\quad$ G.2004.50.3.3, G.2008.34.14.7, G.2011.31.8.3

\author{
Araneae (47): \\ Linyphiidae (1): \\ - G.2004.50.13 \\ Mimetidae (1): \\ - $\quad$ G.2004.50.11 \\ Miturgidae (2): \\ - $\quad$ G.2004.6.2.1, G.2004.50.8.1 \\ Mygalomorpha undetermined (1): \\ - G.2004.50.9.1 \\ Pholcidae (1):
}

- $\quad$ Modisimus sp. G.2004.6.4.1 (Figure 1)

Salticidae (5):

- G.2004.50.3.1, G.2004.50.4.1, G.2004.50.5.1, G.2004.50.12.1, G.2005.147.9

Theraphosidae (1):

- $\quad$ Figured Dunlop et al. (2008, pp. 13-14, figs 2-9) G.2004.6.1.1

Trochanteriidae (4?):

- Veterator sp. G.2004.50.1.1, ?G.2004.50.2.1, ?G.2004.50.10.1 (x2)

Undetermined (31):

- $\quad$ IA.61 (with II.2159 and V.68584), IA.62 (with II.2163 and V.68586), G.1998.54.3.1, G.2004.6.3.1 (x2), G.2004.6.10.1 (x3), G.2004.7.1.1, G.2004.50.3.2, G.2004.50.6, G.2004.50.7.1, G.2005.147.6.1, G.2005.147.7.1, G.2005.147.8, G.2005.147.10, G.2005.147.11.1, G.2006.42.4, G.2006.42.5.1, G.2006.42.7.5, G.2007.67.1.1, G.2007.67.2, G.2007.67.3, G.2007.67.4, G.2007.67.5.4, G.2007.67.14.6, G.2008.34.3.4, G.2008.34.9.3, G.2008.34.13, G.2008.34.14.2, G.2014.50.6.2

Pseudoscorpiones (2):

Atemnidae (1):

- G.2004.6.5.1

Chernetidae (1):

- $\quad$ Figured Ross and Sheridan (2013, p. 54, figure 2) G.2011.31.2.1

MYRIAPODA (5):

Chilopoda (3):

Geophilomorpha (2):

- G.2007.67.10.1, Figured Ross and Sheridan (2013, p. 55, fig. 9) G.2005.147.1.1 (Figure 2)

Scutigeromorpha (1):

- G.2014.50.2 (Figure 3)

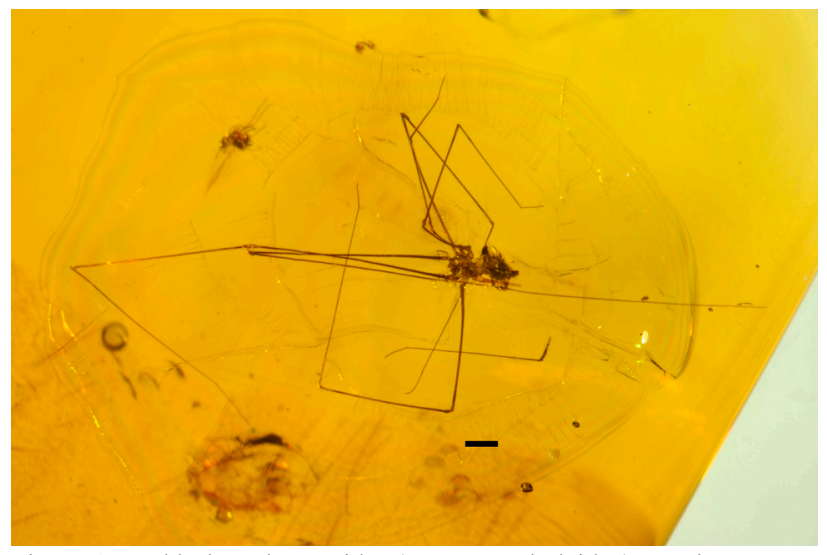

Figure 1. Daddy long-legs spider (Araneae: Pholcidae), Modisimus sp., NMS G.2004.6.4.1. 
Diplopoda (2):

Undetermined (2):

- $\quad$ G.2005.147.2, G.2006.42.2

\section{CRUSTACEA (1):}

Isopoda (1):

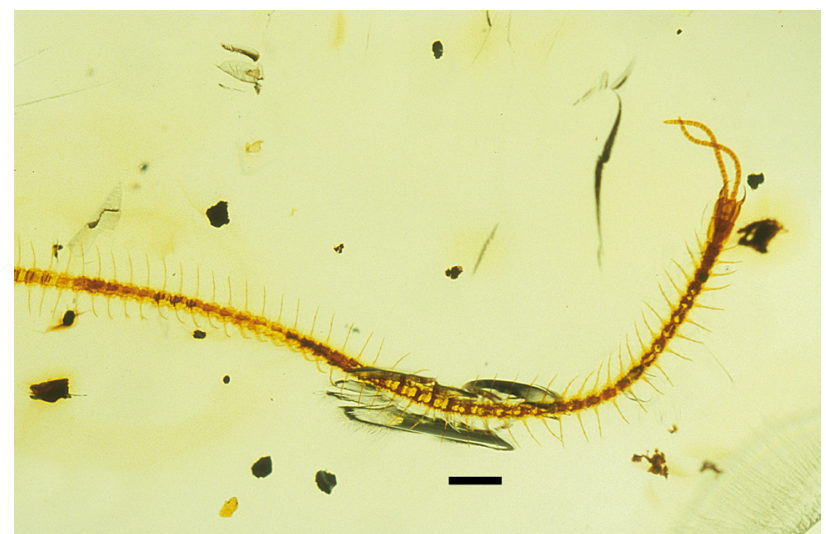

Figure 2. Centipede (Chilopoda: Geophilomorpha), NMS G.2005.147.1.1.

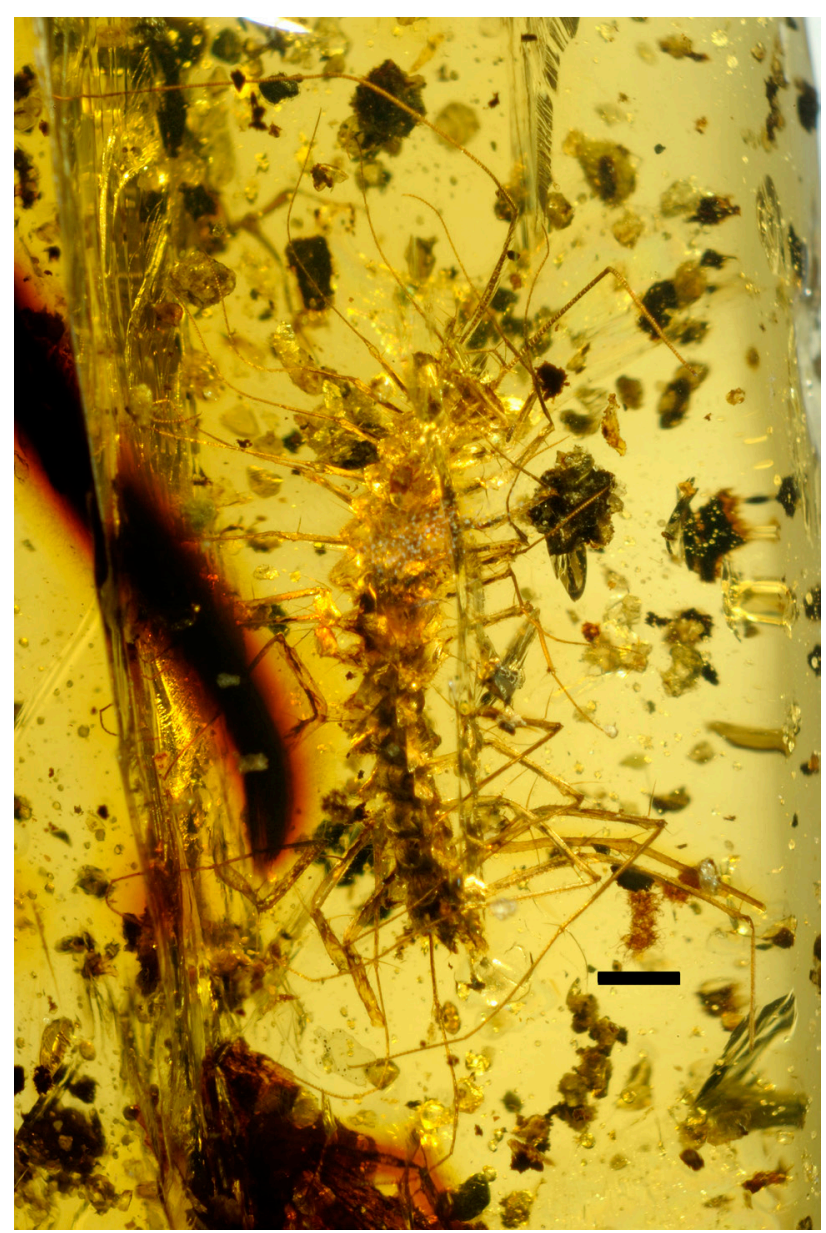

Figure 3. House centipede (Chilopoda: Scutigeromorpha), NMS G.2014.50.2.
Undetermined (1):

- $\quad$ G.2008.34.14.1

\section{HEXAPODA (496):}

Collembola (11):

Undetermined (11):

- II.1863(4), II.2164(2), II.3084(7), G.2004.6.10.3, G.2006.42.3.2, G.2006.42.5.2, G.2008.34.11.5 (x2), G.2014.50.6.3 (x2), G.2014.50.7.3

Archaeognatha (2):

Machilidae (2):

- $\quad$ G.2004.6.6.1, G.2005.147.3.2

Ephemeroptera (3):

Baetidae (1):

- II.2159(1) (Figure 4)

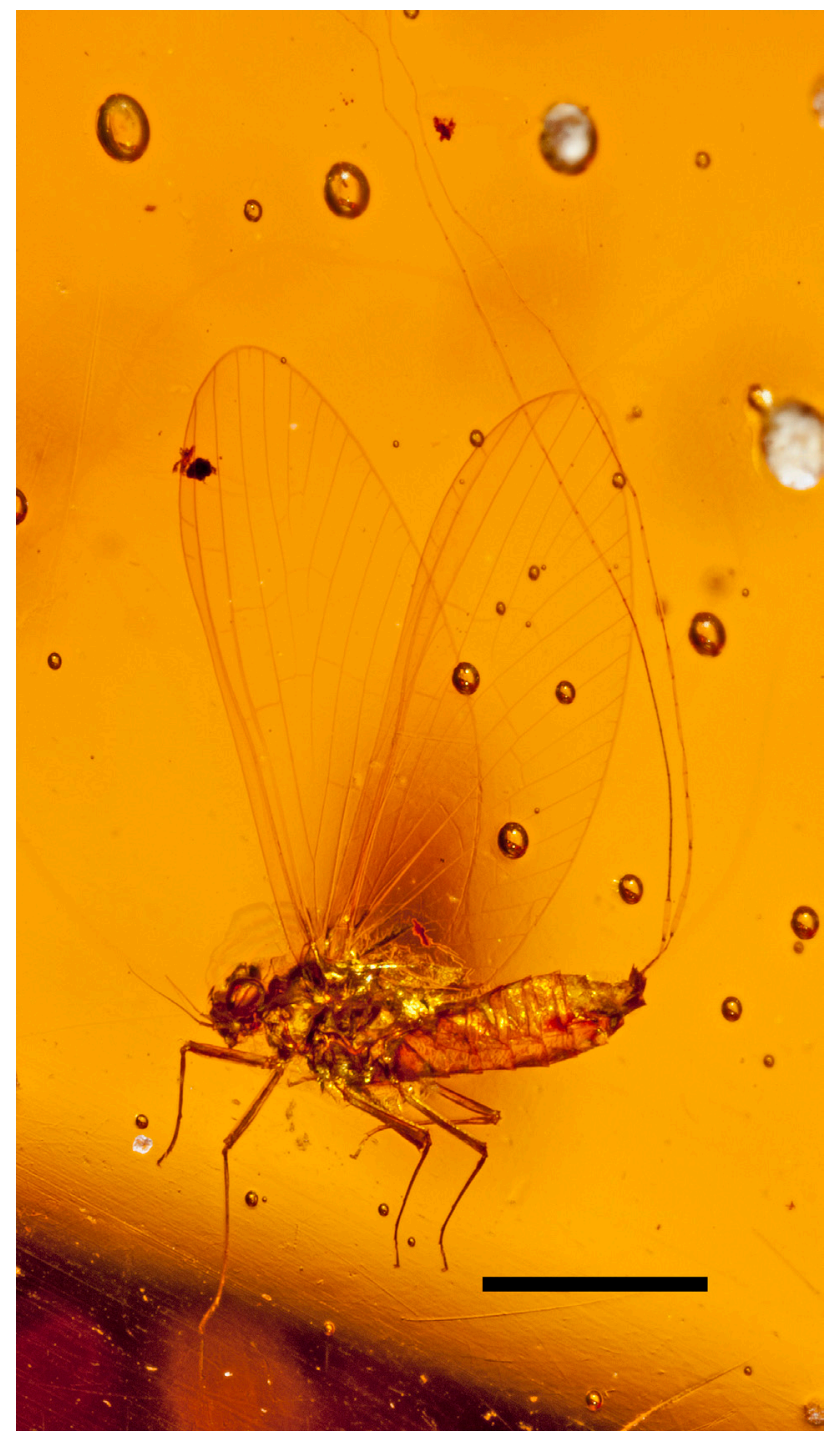

Figure 4. Mayfly (Ephemeroptera: Baetidae), NHM II.2159(1). Photo taken by Harry Taylor (NHM). 


\section{Heptageniidae (2):}

- Maccaffertium annae Macadam and Ross, 2016, Holotype G.2008.34.3.1, Paratype G.2008.34.3.2

\section{Odonata (1):}

Coenagrionidae (1):

- Neoerythromma sp. Figured Ross, Coutiño José and Nel, 2016 G.2008.34.1.1

Blattodea (13):

Ectobiidae (=Blattellidae) (8):

- II.1863(1), II.2165, II.2167(1), G.2014.50.7.1, G.2014.50.8.1

- $\quad$ Anaplecta sp. II.2168(1), G.2011.31.10, Figured Ross and Sheridan (2013,p. 55, fig. 8) G.2006.42.1 (Figure 5)

Undetermined (5):

- $\quad$ II.1862, II.2159(6), G.2004.50.3.4, G.2008.34.11.1, G.2014.50.9.1

\section{Dermaptera (1):}

Diplatyidae (1):

- Haplodiplatys crightoni Ross and Engel, 2013, Holotype G.2011.31.1.1 (Figure 6)

Isoptera (29):

Mastotermitidae (6):

- Mastotermes sp. II.2159(3), G.2005.147.7.2, G.2008.34.6 (x2), G.2014.50.3.1, Figured Ross and Sheridan (2013, p. 54, fig. 3) G.2005.147.4.1

Undetermined (23):

- $\quad$ II.2132(2) (x2), II.2133(1), II.2158(2), II.2160(1), II.2166(2) (x7), G.1998.38.6.1 (x4), G.2000.63.3 G.2005.147.4.2, G.2005.147.5, G.2006.42.6 G.2007.67.11.3, G.2007.67.14.1, G.2008.34.14.3

Orthoptera (2):

Grylloidea undetermined (2):

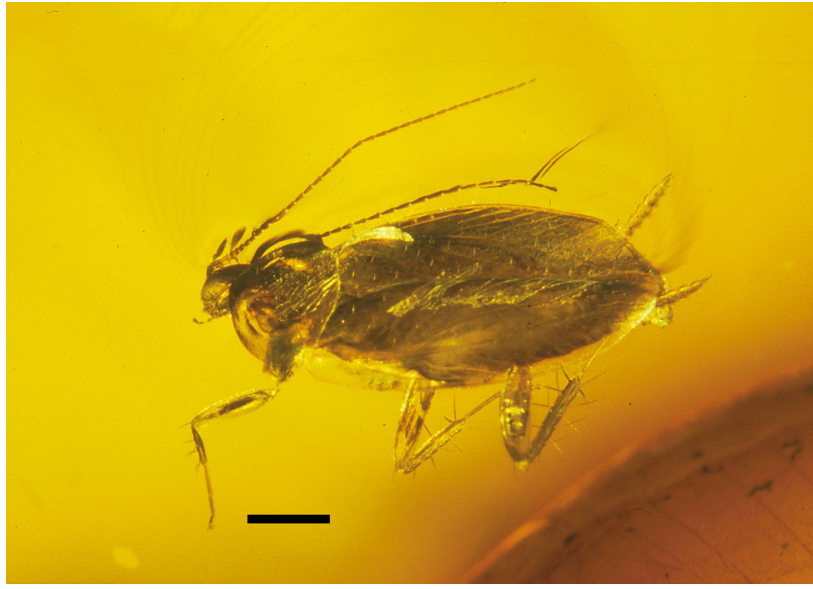

Figure 5. Cockroach (Blattodea: Ectobiidae), Anaplecta sp., NMS G.2006.42.1.

\section{- $\quad$ II.2166(1) (Figure 7), G.2014.50.1 (Figure 8)}

Hemiptera (49):

Aetalionidae (1):

- $\quad$ II.2127(1)

Aleyrodidae (2):

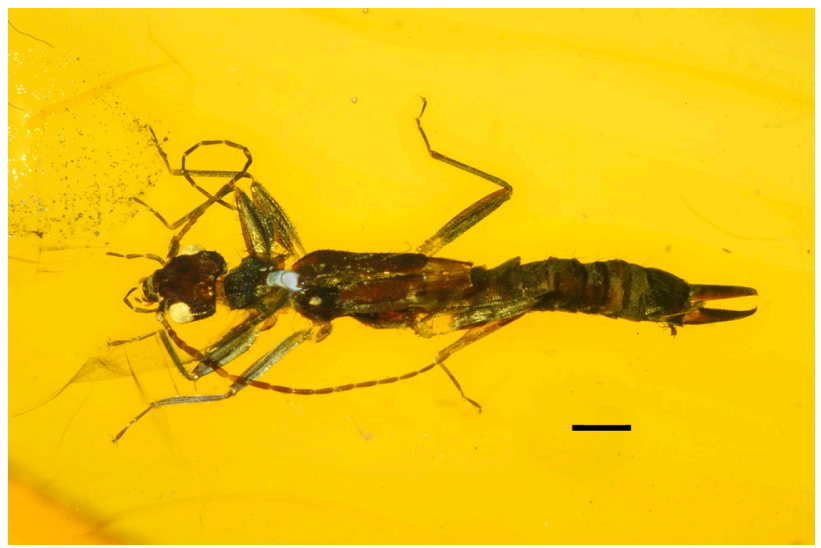

Figure 6. Earwig (Dermaptera: Diplatyidae), Holotype of Haplodiplatys crightoni Ross and Engel, 2013, NMS G.2011.31.1.1.

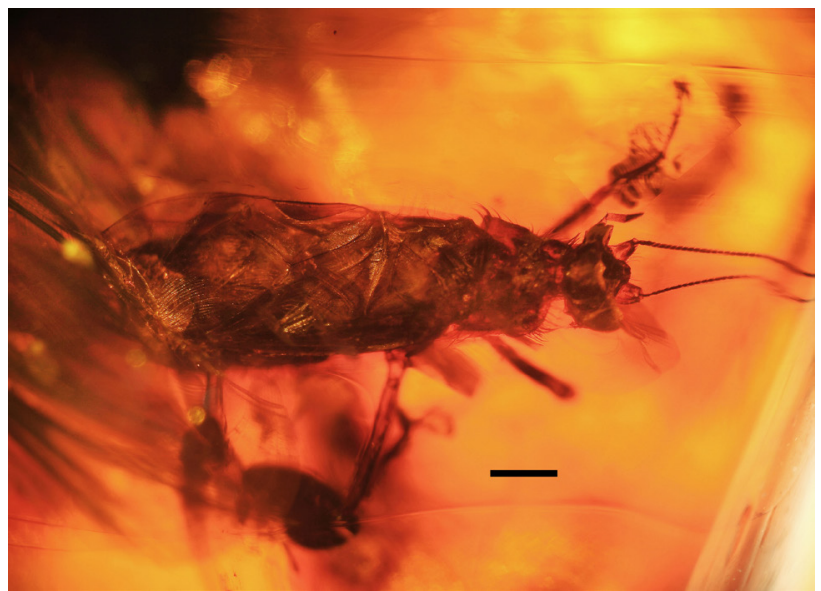

Figure 7. Cricket (Orthoptera: Grylloidea), NHM II.2166(1).

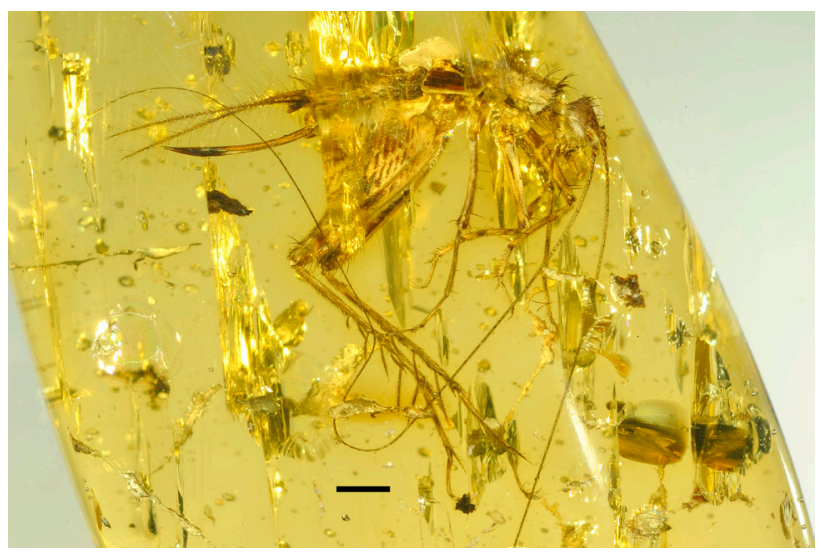

Figure 8. Cricket (Orthoptera: Grylloidea), NMS G.2014.50.1. 
- $\quad$ G.2004.6.10.5, G.2007.67.13.3

Cicadellidae (4):

- G.2004.50.3.5, G.2006.42.7.9, G.2007.67.11.2, Figured Ross and Sheridan (2013, p. 56, fig. 13) G.2008.34.5.1

Cixiidae (6):

- II.2132(1), G.2000.63.1, G.2006.42.7.3, G.2011.31.1.5, G.2011.31.5 (Figure 9), G.2014.50.5.1

Coccoidea undetermined (3?):

- $\quad$ G.2004.50.3.6, G.2008.34.3.8, ?G.2008.34.10.3

Derbidae (1):

- Cedusa baylissae Szwedo and Ross, 2003, Holotype II.2154 (Figure 10)

Fulgoroidea undetermined (14):

- II.2127(2), II.3084(5), G.2004.6.1.2 (x3), G.2004.50.9.3, G.2005.147.6.2, G.2006.42.7.2, G.2008.34.3.3, G.2008.34.10.1, G.2011.31.4.2(x2), G.2014.50.5.2, Figured Ross (1998, fig. 70; 2010, fig. 80) II.1869

Heteroptera undetermined (2):

- $\quad$ II.2133(10), G.2007.67.11.1

Homoptera undetermined (10?):

- II.2168(6), G.2004.6.1.3 (x4), G.2004.6.4.2,

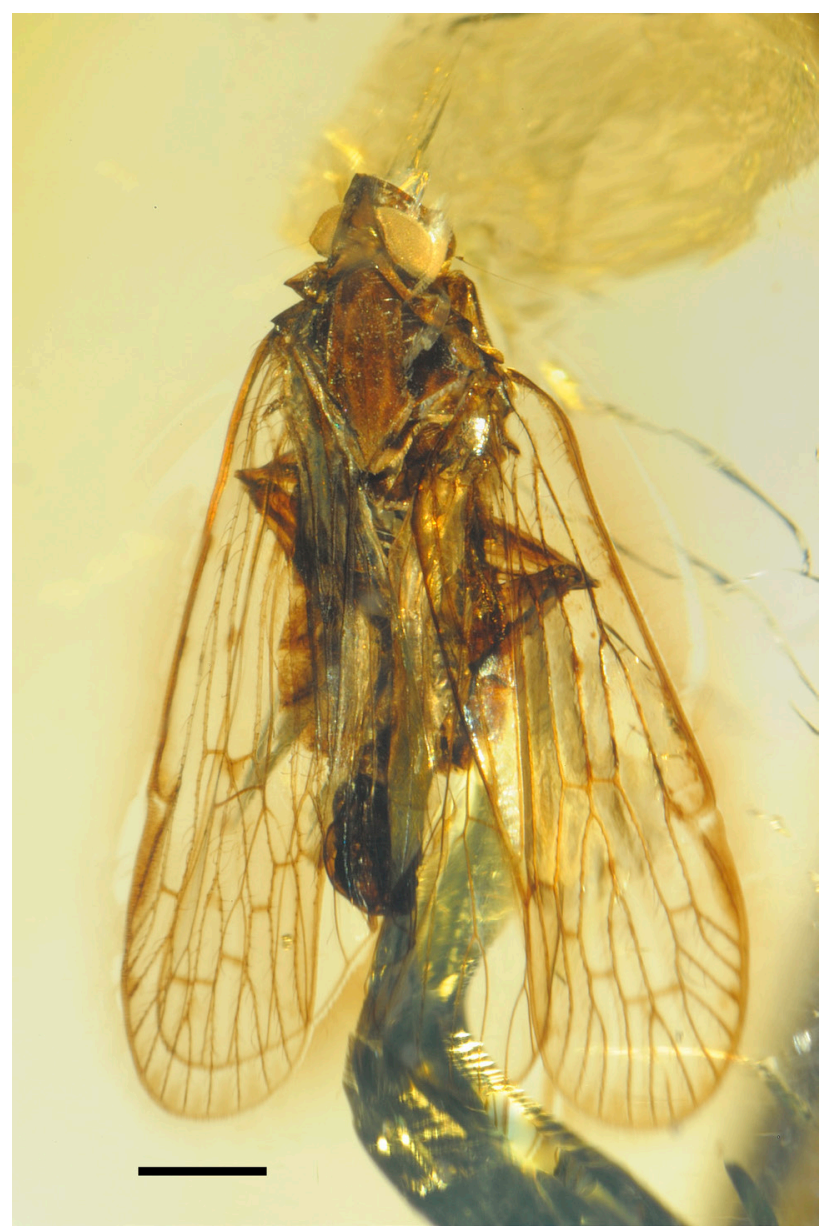

Figure 9. Planthopper (Hemiptera: Cixiidae), NMS G.2011.31.5.
G.2004.50.2.2, ?G.2006.42.5.5, G.2008.34.14.6, G.2014.50.5.7

Kinnaridae (1):

- G.2014.50.6.1 (Figure 11)

Nogodinidae (1):

- Tonacatecutlius gibsoni Stroiński and Szwedo, 2008, Holotype II.2131(1) (Figure 12)

Reduviidae (1):

- $\quad$ Figured Ross and Sheridan (2013, p. 56, fig. 14) G.2008.34.7 (Figure 13)

\section{Tropiduchidae (1):}

- $\quad$ Figured Ross and Sheridan (2013, p. 56, fig. 16) G.2011.31.4.1

Undetermined (2):

- $\quad$ G.2011.31.2.4 (x2)

Psocoptera (13):

Undetermined (13):

- II.1863(3), II.1865(1), II.2132(3), II.3084(6), G.1998.38.6.2, G.2004.6.10.4, G.2004.7.1.2, G.2004.50.3.7 (x2), G.2004.50.7.2, G.2007.67.14.5, G.2014.50.4.2, G.2014.50.5.5

Thysanoptera (5):

Undetermined (5):

- II.2133(2), II.3084(8), G.2007.67.14.10, G.2011.31.7.3 (x2)

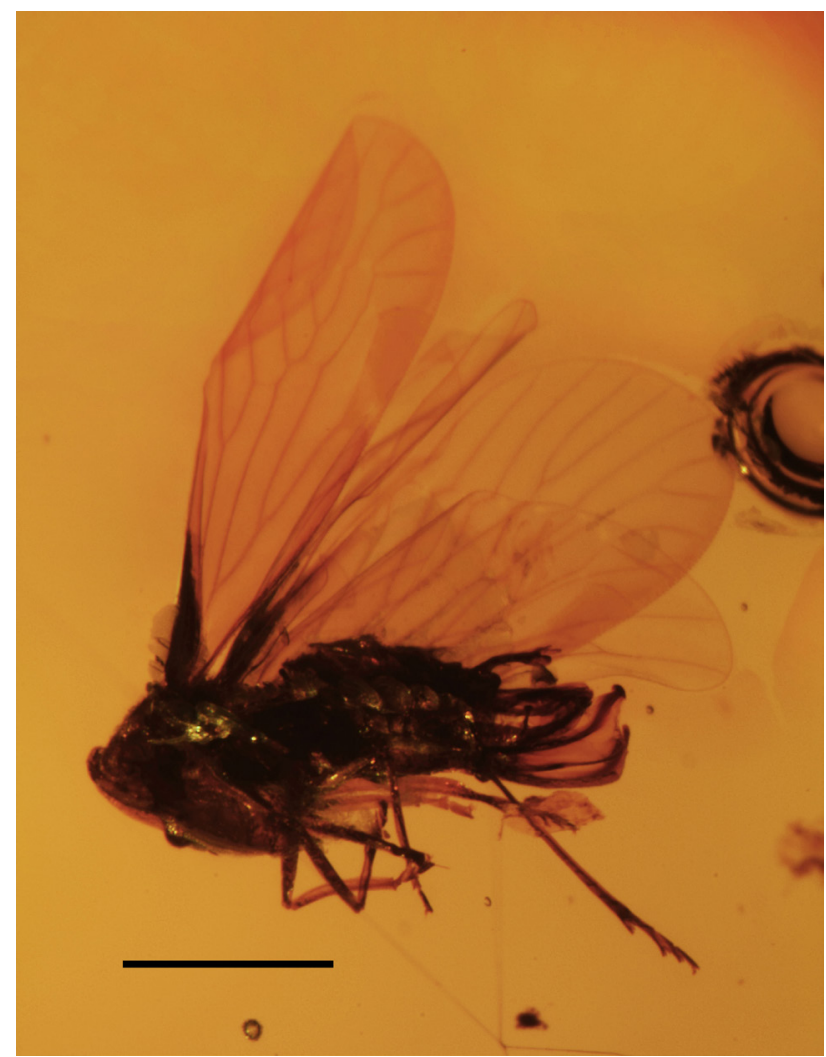

Figure 10. Planthopper (Hemiptera: Derbidae), Holotype of Cedusa baylissae Szwedo and Ross, 2003, NHM II.2154. 


\section{Coleoptera (44):}

Anthribidae (1):

- $\quad$ Figured Ross and Sheridan (2013, p. 55, fig. 6)

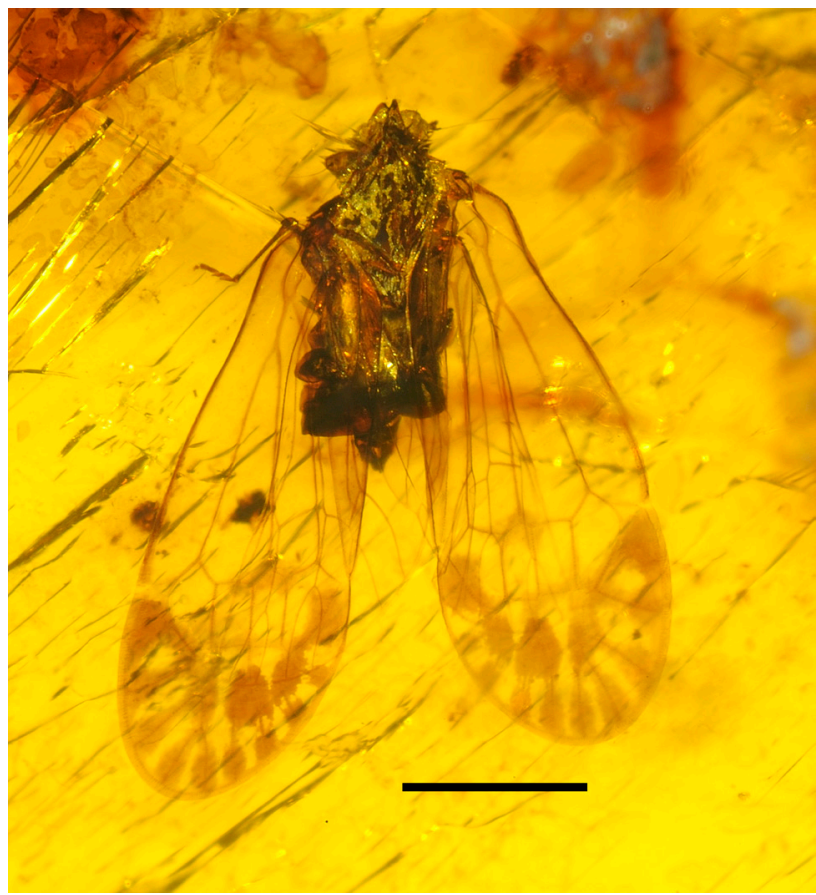

Figure 11. Planthopper (Hemiptera: Kinnaridae), NMS G.2014.50.6.1.

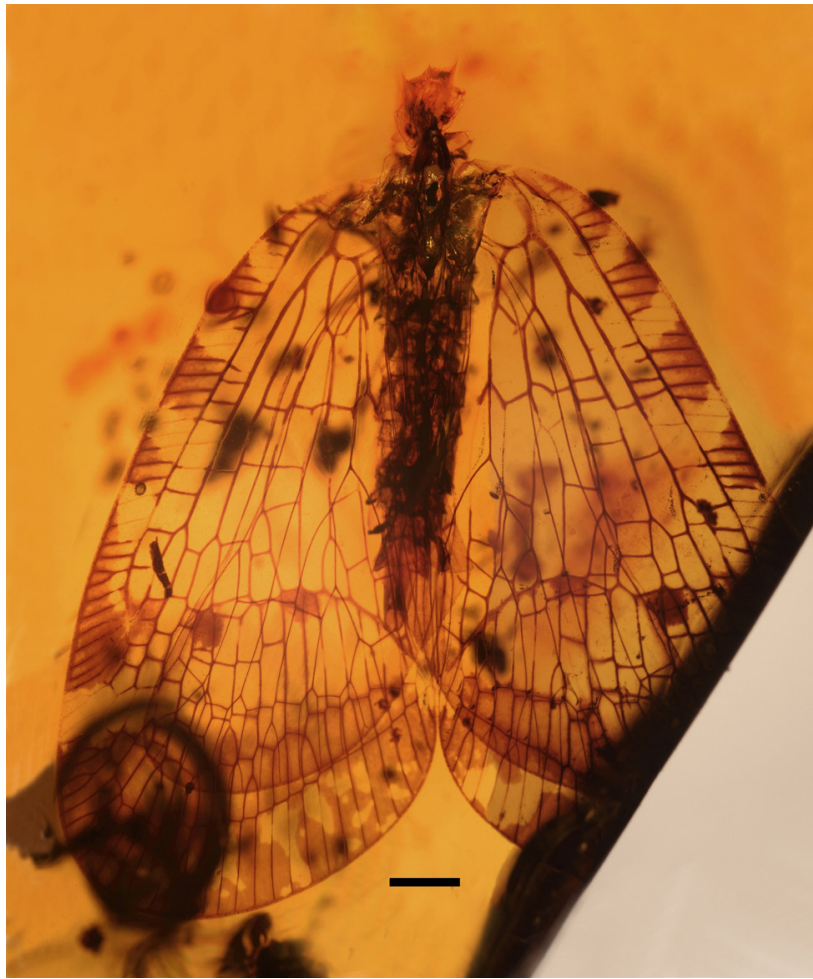

Figure 12. Planthopper (Hemiptera: Nogodinidae), Holotype of Tonacatecutlius gibsoni Stroiński and Szwedo, 2008, NHM II.2131(1).

\section{G.2004.6.8.1 (Figure 14)}

Cerambycidae (2):

- G.2007.67.12.1, Figured Ross and Sheridan (2013, p. 56, fig. 17) G.2008.34.2 (Figure 15)

Curculionidae (2):

- $\quad$ Platypodinae G.2007.67.12.2 (x2)

Curculionoidea undetermined (1):

- II.1863(2)

Dytiscidae (9?):

- $\quad$ Figured Ross and Sheridan (2013, p. 54, fig. 1) ?G.2006.42.3.1 (x9)

Elateroidea undetermined (3):

- $\quad$ II.2164(1), II.3083(1) (Figure 16), G.2008.34.9.1

Staphylinidae (4):

- G.1998.38.6.3, G.2004.50.7.3, G.2007.67.12.3, G.2014.50.9.2

Undetermined (22):

- $\quad$ II.2133(3) (x2), G.1998.38.3.2, G.2004.6.1.4, G.2004.6.9, G.2004.6.10.7 (x6), G.2004.50.3.9, G.2004.50.4.2, G.2004.50.10.2, G.2005.147.9.3, G.2006.42.7.8, G.2007.67.7.1, G.2007.67.12.4 (x2), G.2011.31.2.3, G.2014.50.6.4, G.2014.50.8.3

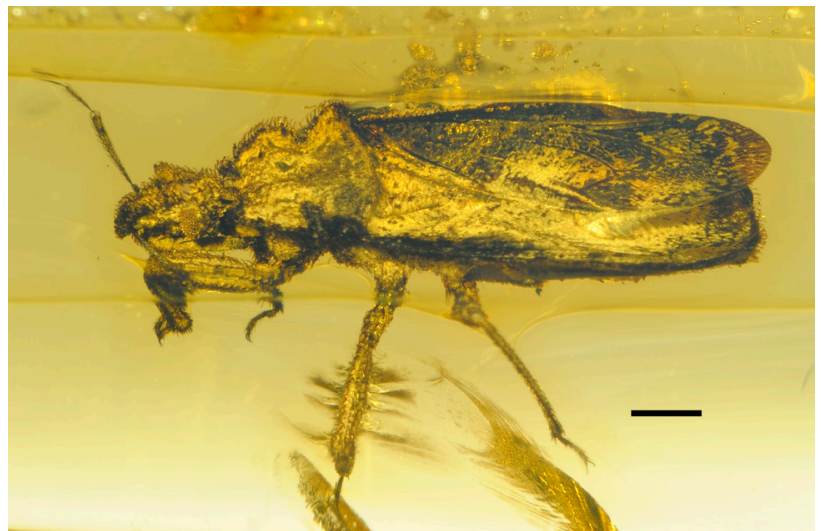

Figure 13. Assassin bug (Hemiptera: Reduviidae), NMS G.2008.34.7.

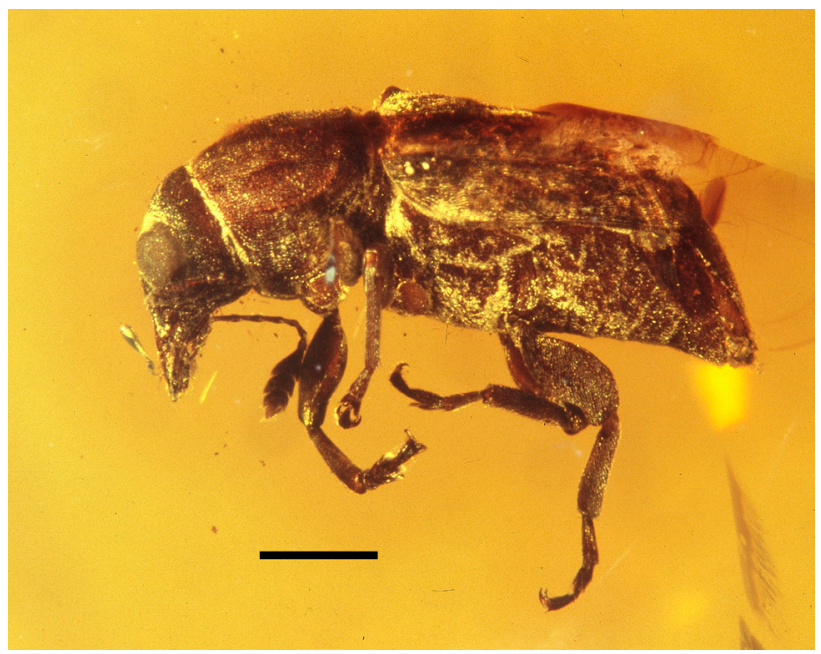

Figure 14. Fungus weevil (Coleoptera: Anthribidae), NMS G.2004.6.8.1. 
Diptera (193):

Cecidomyiidae (63):

- $\quad$ II.1864(2) (x2), II.1865(4), II.2132(5), II.2133(8) (x4), II.2160(2) (x2), II.2168(3) (x4), II.3083(4) (x2), II.3084(3), G.2004.6.1.7, G.2004.6.2.2, G.2004.6.3.3, G.2004.6.10.9 (x4), G.2004.50.3.11 (x6), G.2005.147.9.2, G.2006.42.7.7 (x5), G.2007.67.10.2, G.2007.67.12.6, G.2007.67.14.3, G.2008.34.1.2, G.2008.34.11.3, G.2008.34.14.5, G.2014.50.5.6 (x2), G.2014.50.7.4, G.2014.50.8.5

- $\quad$ Lestremia sp. G.2004.50.1.2 (x6), G.2005.147.6.3, G.2006.42.5.4, G.2007.67.5.3 (x8), G.2011.31.7.2

Ceratopogonidae (5):

- II.1864(3), II.2159(5), II.2168(5). G.2004.6.1.6, G.2014.50.4.6

Chironomidae (25):

- II.1863(6), II.1864(3), II.1865(3), II.1866(2), II.2133(7), II.2166(4), II.2167(3) (x2), II.2168(2) (x4), II.3083(2) (x2), G.2006.42.7.6, G.2007.67.6.3, G.2008.34.3.7 (x2), G.2008.34.11.4, G.2014.50.4.4 (x3), G.2014.50.8.4, G.2014.50.9.3 (x2)

Culicidae (2):

- $\quad$ G.2004.6.1.5 (x2)

Cyclorrapha undetermined (2):

- $\quad$ II.2166(5), G.2007.67.10.3

Dolichopodidae (9):

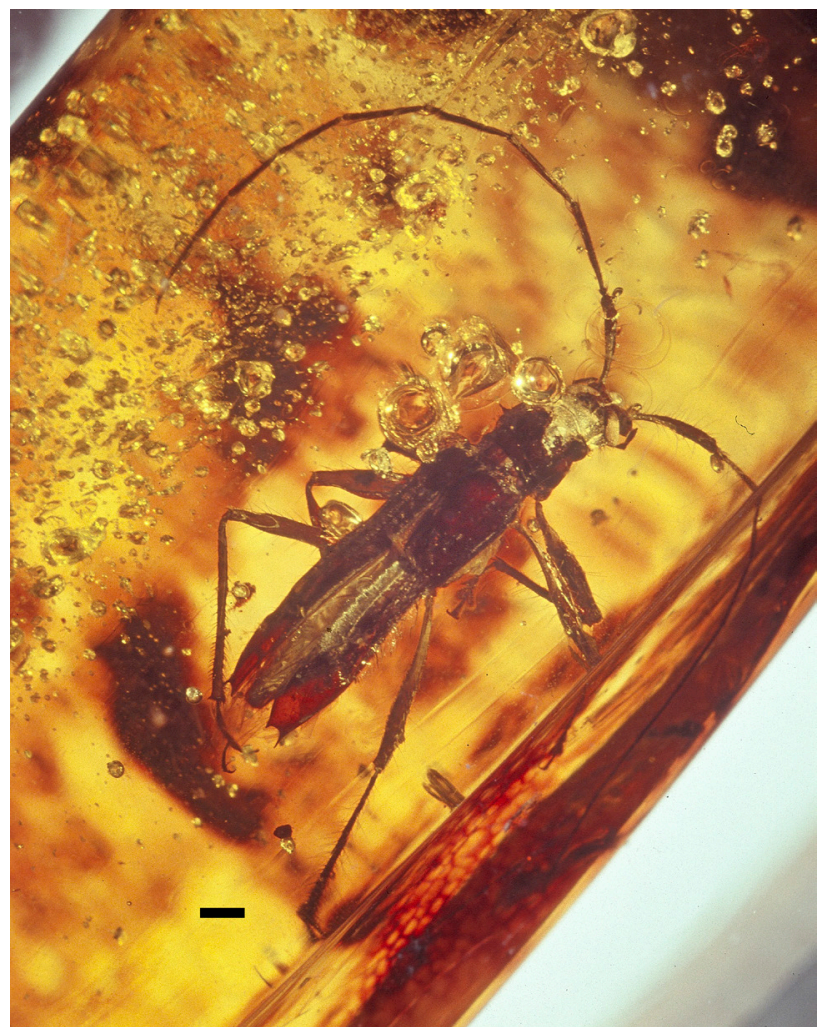

Figure 15. Long-horn beetle (Coleoptera: Cerambycidae), NMS G.2008.34.2
- $\quad$ II.1871, II.2168(9), G.1998.38.6.5, G.2004.6.3.2, G.2004.6.10.10, G.2007.67.14.8, G.2011.31.9.1 (x3)

Drosophilidae (1):

- II.1864(5)

Keroplatidae (2):

- $\quad$ G.2004.50.3.10, G.2007.67.14.2

Limoniidae (2):

- $\quad$ G.2000.63.2

- Toxorhina sp. II.1870

Mycetophilidae (2):

- $\quad$ II.1866(1) (Figure 17), G.2004.7.1.3

Nematocera undetermined (7):

- $\quad$ G.2004.7.1.4 (x2), G.2007.67.12.5, G.2007.67.14.9 (x2), G.2014.50.9.4 (x2)

Phoridae (22):

- II.894(1), II.895(1), II.2133(9), II. 2159(2), II.2163 (x11), II.3084(4), G.2004.50.3.12 (x2), G.2004.50.5.2 G.2008.34.5.2 (x3)

Psychodidae (25):

- II.894(2), II.1865(2), II.2133(12), II.2160(3), II.2168(8), G.2004.6.4.3 (x3), G.2004.6.10.8 (x6), G.2006.42.5.3 (x2), G.2007.67.14.7, G.2008.34.11.2, G.2008.34.14.4, G.2011.31.1.3, G.2014.50.4.3 (x4)

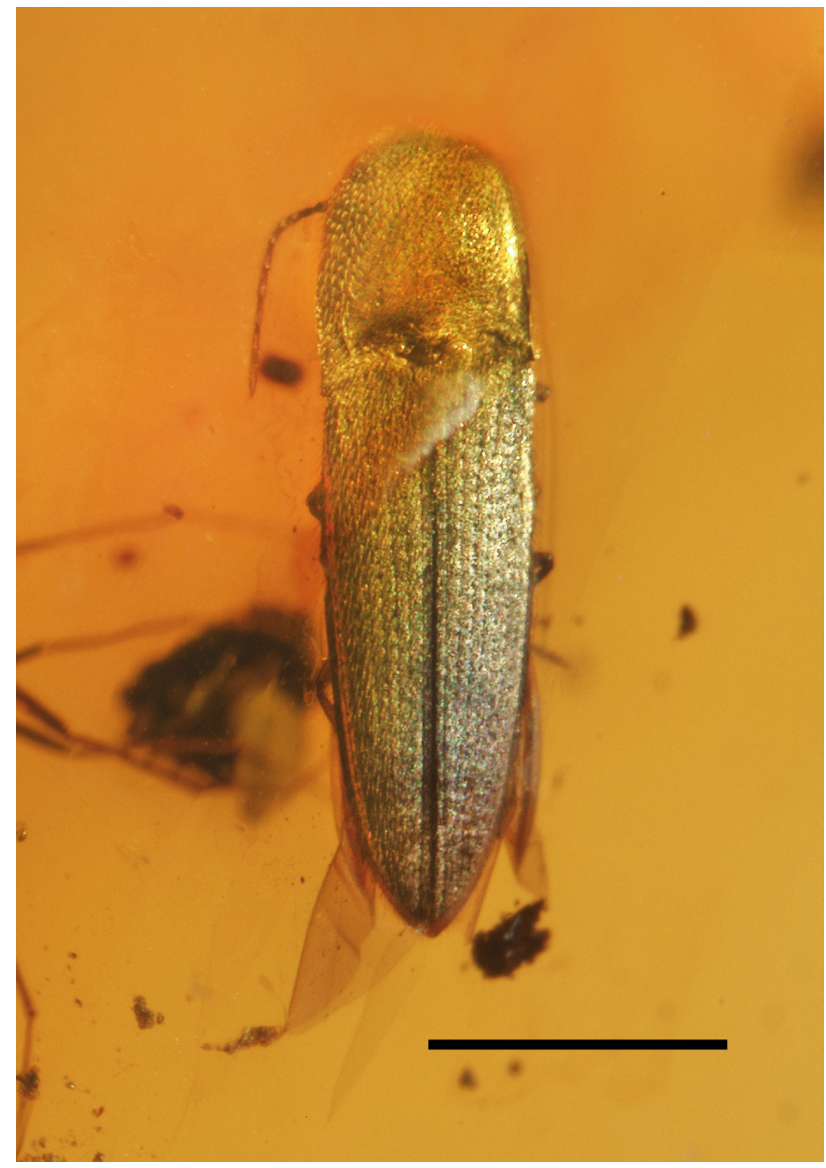

Figure 16. Click beetle (Coleoptera: Elateroidea), NHM II.3083(1). 


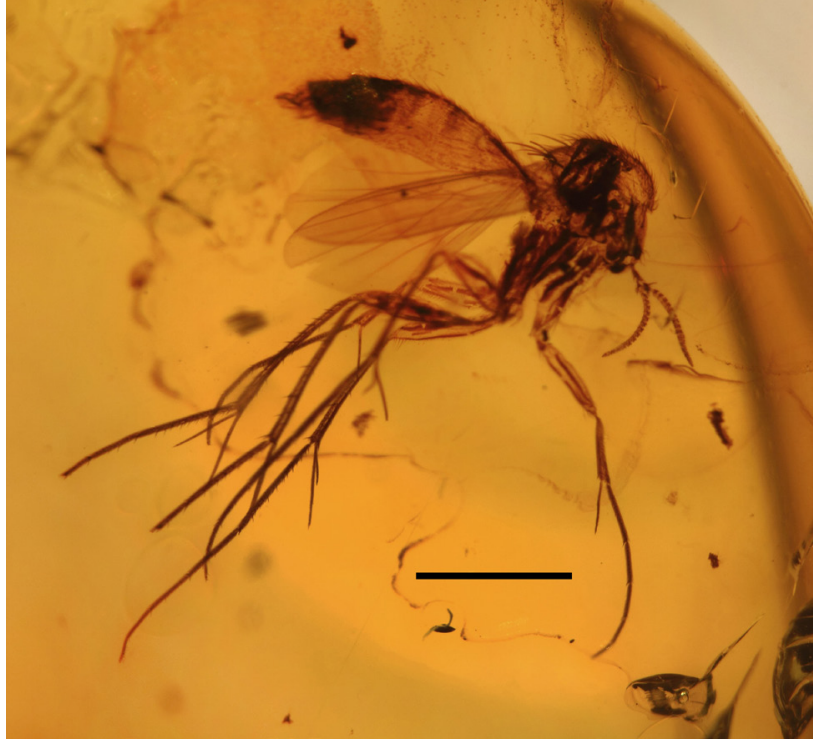

Figure 17. Fungus gnat (Diptera: Mycetophilidae), NHM II.1866(1).

- $\quad$ Phlebotominae II.1863(7)

Rhagionidae (1):

- $\quad$ Figured Ross and Sheridan (2013, p. 55, fig. 4) G.2008.34.4

Scatopsidae (6):

- II.1867(1), II.3083(3) (x2), G.1998.38.6.4, G.2007.67.1.2, G.2014.50.4.5

Sciaridae (7):

- $\quad$ II.1865(5), II.2133(11), II.2168(4), G.2004.6.10.11, G.2007.67.6.2, G.2007.67.14.4, G.2014.50.3.2

Sciaroidea undetermined (2):

- $\quad$ II.2166(3), G.2007.67.13.2

Stratiomyidae (1):

- $\quad$ G.2008.34.12 (Figure 18)

Tephritidae (1):

- $\quad$ II.2169 (Figure 19)

Therevidae (1):

- Peratrimera mexicana Hauser and Irwin, 2005, Holotype II.2158(1) (Figure 20)

Tipuloidea undetermined (3)

- $\quad$ G.2005.147.3.3, G.2007.67.6.1, G.2008.34.9.2

Undetermined (4)

- $\quad$ G.2004.6.10.12 (x2), G.2011.31.4.3, G.2011.31.7.1

Hymenoptera (97):

Apidae (14):

- $\quad$ Meliponini II.3084(1) (x3), G.2004.50.1.3 (x6), G.2004.50.2.3, G.2005.147.11.2, G.2011.31.9.2 (x2), G.2014.50.5.3

Apocrita undetermined (3):

- $\quad$ G.2007.67.5.2, G.2014.50.4.7 (x2)

Bethylidae (1):

- $\quad$ II.2162 (Figure 21)

Braconidae (2):

- $\quad$ II.1868 (Figure 22), G.2011.31.1.2

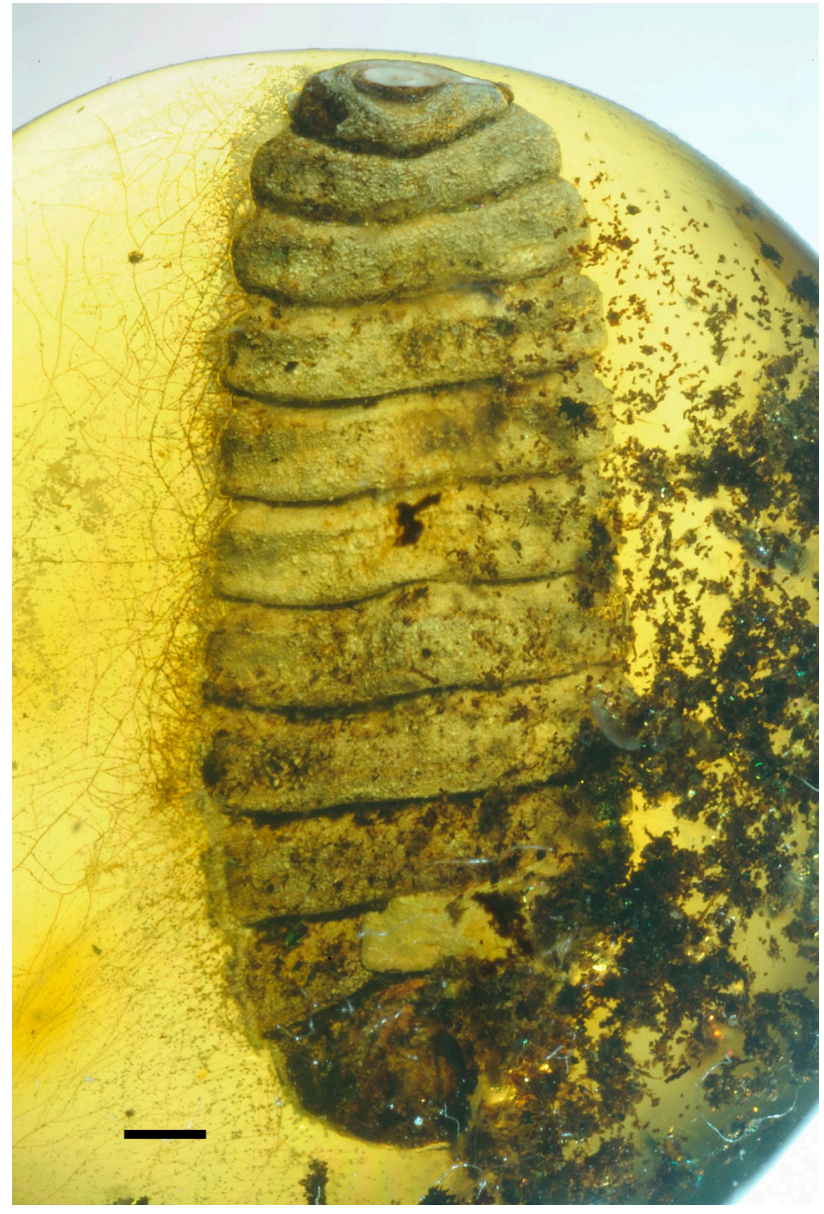

Figure 18. Soldier fly larva (Diptera: Stratiomyidae), NMS G.2008.34.12.

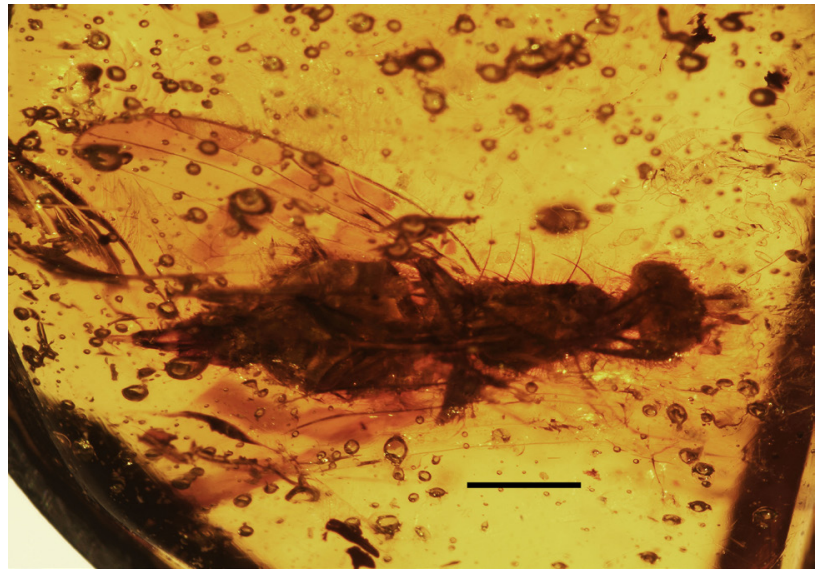

Figure 19. Fruit fly (Diptera: Tephritidae), NHM II.2169.

Chalcidoidea undetermined (3):

- $\quad$ II.2168(7), G.2004.50.3.14, G.2008.34.3.5

Formicidae (34):

- II.895(2), II.1867(2), II.2131(2), II.2159(4), II.3084(2) (x3), G.1998.38.3.3, G.1998.38.6.6, G.1998.54.3.3, G.2004.6.6.2, G.2004.6.8.2, G.2004.6.10.2 (x4), G.2004.7.1.6 (x3), 


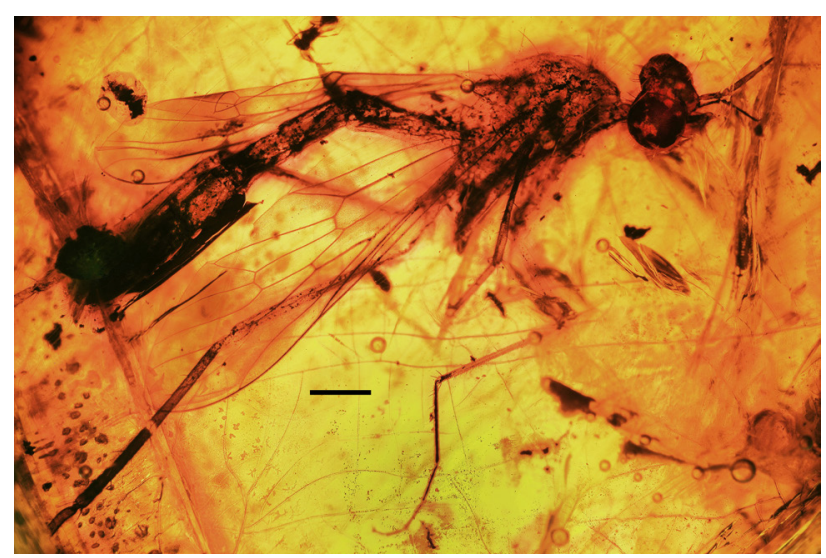

Figure 20. Stiletto fly (Diptera: Therevidae), Holotype of Peratrimera mexicana Hauser and Irwin, 2005, NHM II.2158(1).

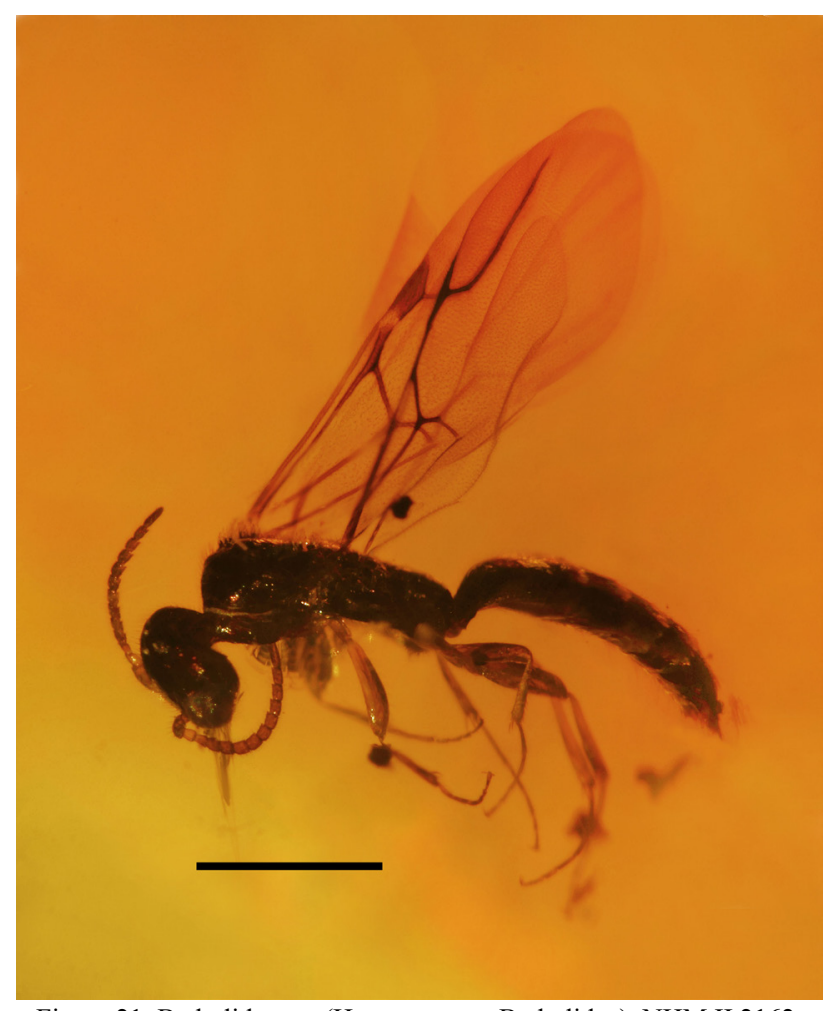

Figure 21. Bethylid wasp (Hymenoptera: Bethylidae), NHM II.2162.

G.2004.50.4.3, G.2004.50.9.4, G.2004.50.12.2, G.2005.147.1.2, G.2007.67.9 (x5), G.2008.34.1.4, G.2008.34.10.2, G.2011.31.2.2, G.2011.31.8.2, G.2014.50.5.4, G.2014.50.7.2

Ichneumonoidea undetermined (1):

- Figured Ross and Sheridan (2013, front cover, p. 6) G.1998.54.3.2

Mymaridae (6):

- II.2133(5) (x2), G.2004.6.3.4, G.2004.7.1.7, G.2004.50.3.15, G.2004.50.7.5

Platygastroidea undetermined (1):

- $\quad$ II.2133(4)

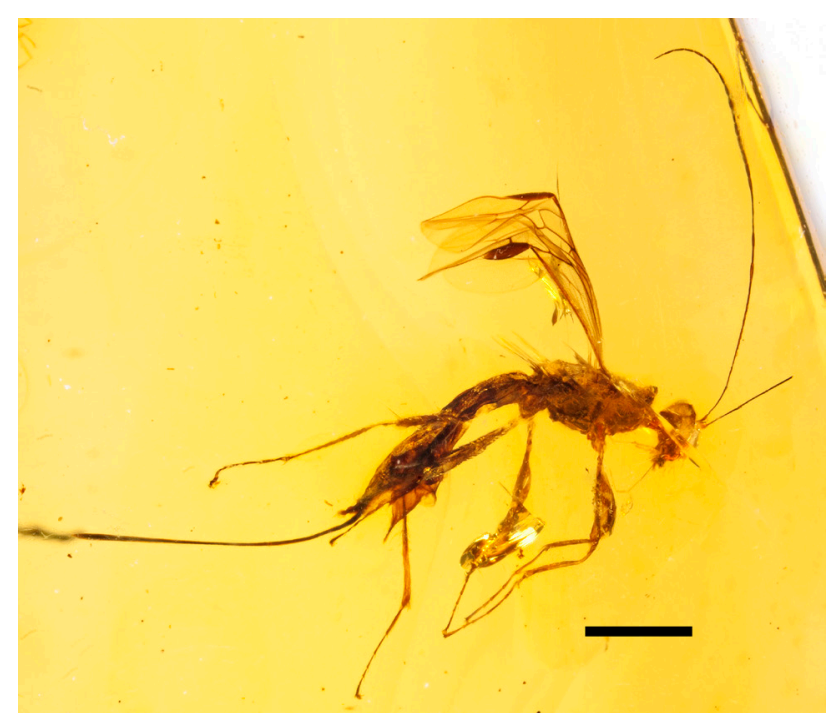

Figure 22. Braconid wasp (Hymenoptera: Braconidae), NHM II.1868. Photo taken by Harry Taylor (NHM).

Scelionidae (1?):

- ?II.1872

Parasitica undetermined (31):

- II.1863(5), II.2132(4), II.2133(6), II.2158(3), II.2167(2), II.3083(5), G.2004.6.10.13 (x4), G.2004.7.1.5 (x4), G.2004.50.1.4 (x2), G.2004.50.3.13, G.2004.50.7.4, G.2005.147.3.4, G.2005.147.9.4, G.2006.42.7.4 (x7), G.2008.34.1.3, G.2008.34.3.6, G.2011.31.1.4 (x2)

Lepidoptera (10):

Undetermined (10):

- II.2161, G.2004.6.10.6, G.2004.50.3.8, G.2004.50.9.2, G.2006.42.7.1, G.2007.67.5.1, G.2007.67.13.1, G.2011.31.6, G.2014.50.8.2,

- Figured Ross and Sheridan (2013, p. 56, fig. 18) G.2008.34.8

Trichoptera (10):

Glossosomatidae (1):

- II.1864 (6)

Hydropsychidae (4):

- Plectropsyche alvarezi Wichard, Solórzano Kraemer and Luer, 2006 (det. Wilfried Wichard) II.1864(1) (x4) (Figure 23)

Undetermined (5):

- $\quad$ G.2008.34.3.9, G.2014.50.4.1 (x4)

Undetermined larvae (16):

- $\quad$ G.2004.6.5.2 (x14), G.2007.67.7.2, G.2007.67.8

Insect coprolites:

- G.2007.67.15 


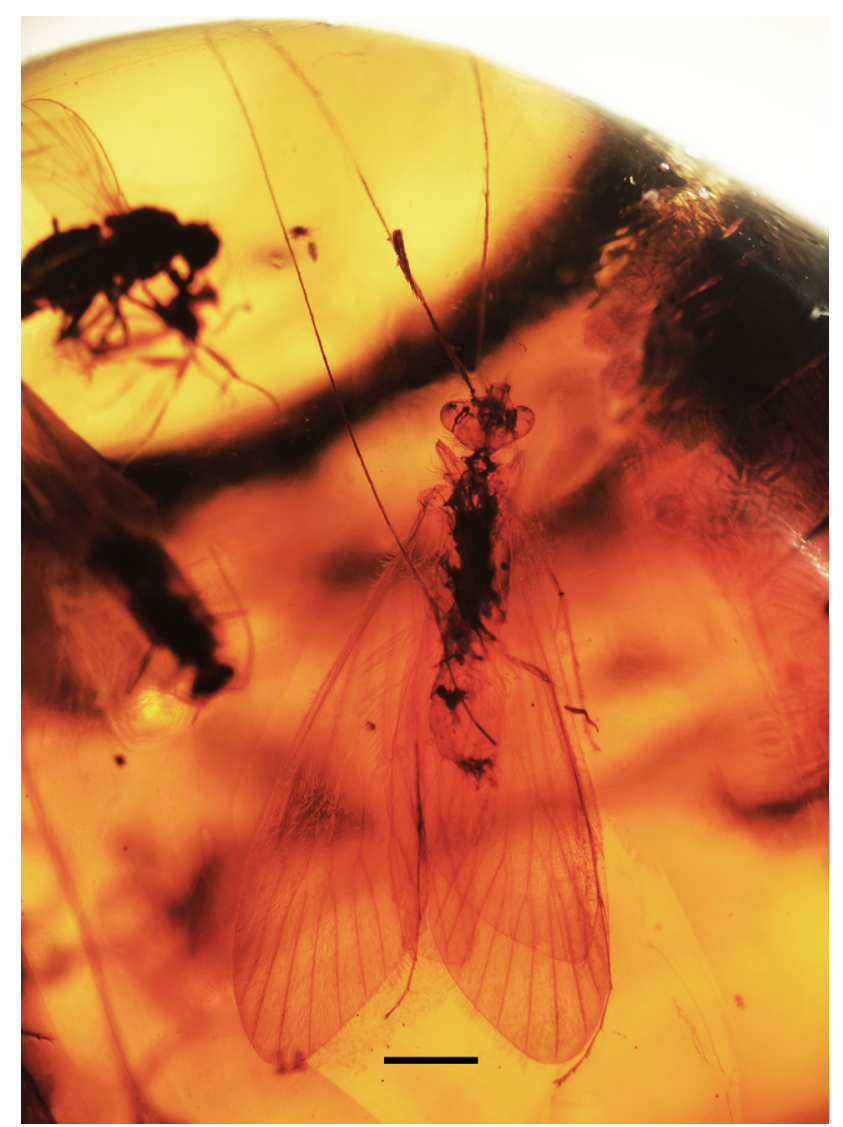

Figure 23. Caddisfly (Trichoptera: Hydropsychidae), Plectropsyche alvarezi Wichard, Solórzano Kraemer and Luer, 2006, NHM II.1864(1).

\section{MOLLUSCA:}

Bivalvia:

Pholadidae borings

- G.2011.31.14

\section{PLANTAE (13?):}

Marchantiophyta (1):

Jungermanniales (1):

Lejeuneaceae (1?):

- Figured Ross and Sheridan (2013, p. 55, fig. 11) ?G.1998.38.3.1

Angiospermae (8):

Ericales (1):

Ericaceae (1?):

- $\quad$ Figured Ross and Sheridan (2013, p. 55, fig. 7) ?G.2004.6.7

Undetermined (7):

- V.68584 (with IA.61 and II.2159), V.68585 (with II.3084), V.68586 (with IA.62 and II.2163), G.2004.50.5.3, G.2005.147.3.1, G.2011.31.8.1, Figured Solórzano Kraemer (2010, fig. 8A), Ross and Sheridan (2013, p. 56, fig. 12) G.2007.67.16

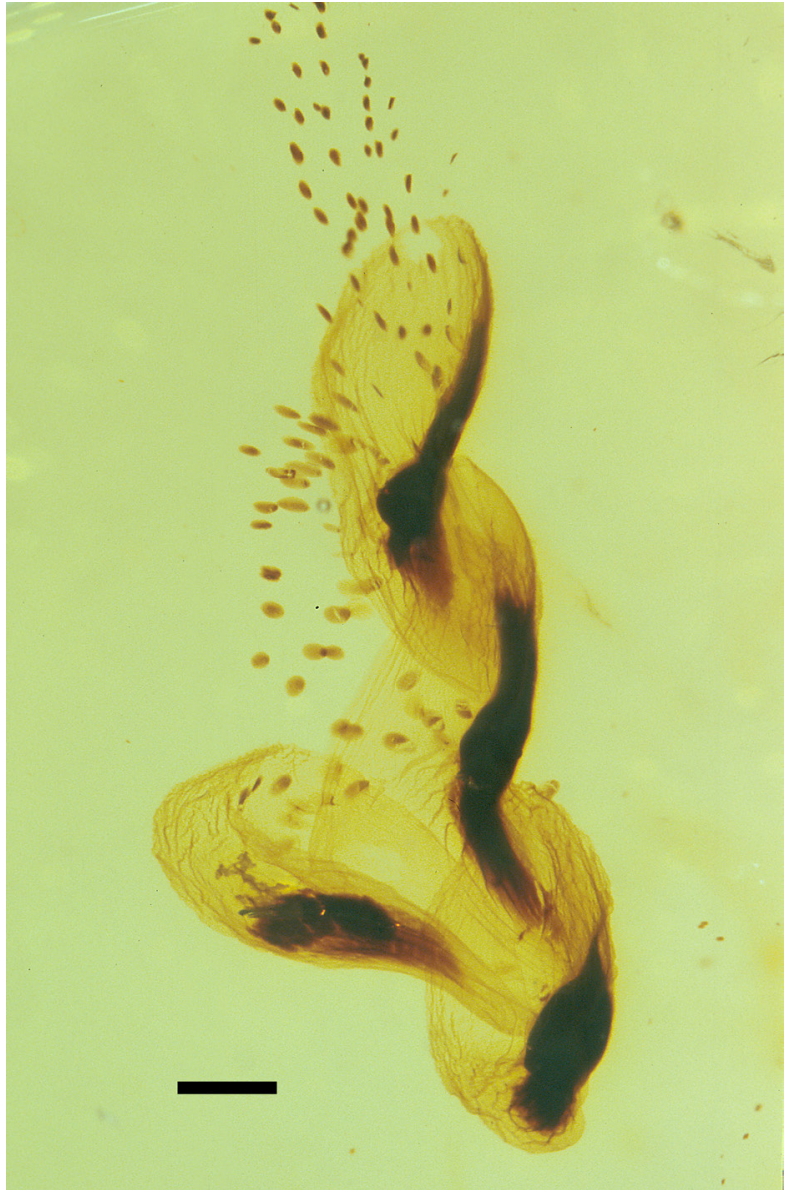

Figure 24. Undetermined plant or fungal structure shedding spores, NMS G.2008.34.15.

Coniferae (1):

Undetermined (1):

- $\quad$ G.2008.34.16

Undetermined (3):

- $\quad$ G.2008.34.17, G.2011.31.3, G.2014.50.7.5

Plantae or Fungi undetermined (1):

- $\quad$ Figured Ross and Sheridan (2013, p. 56, fig. 15) G.2008.34.15 (Figure 24)

\section{Acknowledgements}

Many thanks to Harry Taylor (NHM) for photographing the mayfly in Figure 4, and to Vicen Carrio for writing the Spanish translation of the abstract.

\section{References}

Dunlop, J.A., Harms, D., Penney, D., 2008, A fossil tarantula (Araneae: Theraphosidae) from Miocene Chiapas amber, Mexico: Revista Ibérica de Aracnología, 15, 9-17. 
Hauser, M., Irwin, M.E., 2005, A new remarkable Xestomyzinae (Insecta, Diptera, Therevidae) genus from Mexican amber: Zootaxa, 1008, 39-45.

Macadam, C.R., Ross, A.J., 2016, A New Species of Mayfly, Maccaffertium annae sp. n. (Ephemeroptera: Heptageniidae) from Mexican Amber (Miocene). Boletín de la Sociedad Geológica Mexicana, this volume.

Ross, A.J., Coutiño José, M.A., Nel, A., 2016, The first records of coenagrionid damselflies (Odonata: Zygoptera: Coenagrionidae: Neoerythromma sp. and Nehalennia sp.) from Mexican Amber (Miocene). Boletín de la Sociedad Geológica Mexicana, this volume.

Ross, A.J., 1998, Amber, The Natural Time Capsule: London, UK, Natural History Museum, $73 \mathrm{p}$.

Ross, A.J., 2010, Amber, The Natural Time Capsule, $2^{\text {nd }}$ Edition: London, UK, Natural History Museum. 112 p.

Ross, A.J., Engel, M.S., 2013, The first diplatyid earwig in Tertiary amber (Dermaptera: Diplatyidae): A new species from Miocene Mexican amber: Insect Systematics \& Evolution, 44, 157-166.

Ross, A.J., Sheridan, A., 2013, Amazing Amber: Edinburgh, UK, NMS Enterprises Limited - Publishing. 64 p.
Solórzano Kraemer, M.M., 2010, Mexican amber, in Penney, D. (ed.), Biodiversity of fossils in amber from the major world deposits: Manchester, UK, Siri Scientific Press, 42-56.

Stroiński, A., Szwedo, J., 2008, Tonacatecutlius gibsoni gen. and sp. nov. from the Oligocene/Miocene Mexican amber (Hemiptera: Fulgoromorpha: Nogodinidae): Annales Zoologici, 50, 341-345.

Szwedo, J., Ross, A.J., 2003, Cedusa baylissae sp. nov. from Oligocene/ Miocene Mexican amber (Hemiptera: Fulgoromorpha: Derbidae): Annals of the Upper Silesian Museum (Entomology), 12, 37-46.

Wichard, W., Solórzano Kraemer, M.M., Luer, C., 2006, First caddisfly species from Mexican amber: Zootaxa, 1378, 37-48.

Manuscript received: November 14, 2014.

Corrected manuscript received: March 27, 2015.

Manuscript accepted: April 5, 2015. 